\title{
PlayCubes: Monitoring Constructional Ability in Children using a Tangible User Interface and a Playful Virtual Environment
}

\author{
Sigal Jacoby, Galia Gutwillig, Doron Jacoby, Naomi \\ Josman and Patrice L. (Tamar) Weiss \\ Department of Occupational Therapy \\ University of Haifa \\ Haifa, ISRAEL \\ sigal.jacoby@gmail.com
}

\author{
Minori Koike, Yuichi Itoh, Norifumi Kawai and \\ Yoshifumi Kitamura \\ Human Interface Engineering Laboratory, Graduate \\ School of Information Science and Technology, Osaka \\ University, Osaka, JAPAN \\ \{itoh, kitamura\}@ist.osaka-u.ac.jp
}

\author{
Ehud Sharlin \\ Interactions Laboratory, Department of Computer Science, \\ University of Calgary \\ Calgary, Alberta, CANADA \\ ehud@cpsc.ucalgary.ca
}

\begin{abstract}
Constructional ability is a necessary part of everyday functioning for adults and children alike. In children, the need for adequate levels of constructional ability is evident during both recreational and school activities. PlayCubes is a dynamic tool that supports monitoring a variety of the perceptual-motor processes needed while children perform constructional tasks. PlayCubes is based on ActiveCube (AC), an electronic, Lego-like tangible user interface (TUI) designed to construct threedimensional (3D) objects in both the physical and virtual realms simultaneously. In this paper we explore PlayCubes' use as a concrete, ecologically valid tool which reveals the dynamic functional processes underlying constructional ability among typically developed children. Our paper describes the PlayCubes interface including its interactive playground virtual setting, the motivation for the study, the experimental design and results to date. We conclude that, despite several technical limitations, the PlayCubes system and playground apparatus tasks appear to be sensitive to differences in children constructional abilities while presenting an engaging and enjoyable assessment tool.
\end{abstract}

Keywords - Tangible User Interfaces; constructional ability; playcubes

\section{INTRODUCTION}

Constructional ability entails visuospatial perception with appropriate motor responses [1]. It is a necessary skill for participation in daily life activities. For example, people with visuospatial deficits may have problems in carrying out mathematical calculations, route finding and map reading, drawing and copying diagrams and assembling objects from parts [2]. Moreover, there is ample evidence that impaired performance on constructional tests predicts limitations in important activities such as meal planning and driving [1]. Constructional ability is a basic need for most activities that preschool and school age children perform such as play since

This research was supported in part by Special Coordination Funds of the Science and Technology Agency of the Japanese Government, by a Canadian NSERC Discovery Grant, and by the University of Haifa Development Fund. many games require constructional ability (e.g., building blocks, puzzle assembly, drawing). Such activities provide opportunities to learn and practice fine motor skills, to increase social awareness and to advance cognitive skills.

Constructional activities may be divided into two large classes including drawing and assembling or constructing [1]. In the first class there are two major type of drawing: copying and free drawing. The second class includes assembling tasks (e.g., puzzles) or building tasks which may be two or three dimensional (3D). Spatial perception is a critical component for all these tasks.

Difficulties in constructional performance are typically assessed via observation of a child's ability to construct a spatial model that is presented to him. The outcome measures are related to the time required to complete the task, the accuracy of the finished structure, the types of errors and, to a lesser extent, the strategy used during construction [1]. Twodimensional constructional assessment tests, such as the Block Design and Object Assembly sub tests from the Wechsler intelligence scales are common (WPPSI, Wechsler Preschool and Primary Scale of Intelligence; WISC, Wechsler Intelligence Scale for Children; WAIS, Wechsler Adults Intelligence Scale) [3-5]. Three-dimensional constructional assessment tests are far less common.

Fisher \& Loring [1] described several 3D constructional ability assessment tests including the Test of ThreeDimensional Block Construction and the Block Model. Both these tests require the participant to use a set of wooden blocks to copy and match a $3 \mathrm{D}$ prototype. They also indicated the potential use of children's building toys such as Lego blocks, K'nex and the Tinker Toys for similar assessment tasks, even though, with the exception of Tinker Toys, they do not have a standard assessment procedure. In pediatric occupational 
therapy some tests of cognitive ability include items that assess constructional abilities, for example in the visuomotor construction domain of the Dynamic Occupational Therapy Cognitive Assessment for children (DOTCA-Ch) [6].

There has been much recent research to show the importance of presenting participants with stimuli that provide natural opportunities to behave and, at the same time, probe this behavior using measures that are both reliable and ecologically valid [7-10]. Paper-and-pencil tests and some of their parallel computerized assessments often offer limited stimuli which map poorly to real world tasks.

In addition to their readily apparent advantages of automation and objectivity, virtual displays offer the ability to provide ecologically valid stimuli that assess participant performance in a simulated environment which is dynamic, believable and realistic. Furthermore, testing in virtual environments can potentially reduce testing biases and enhance reliability [11-12].

PlayCubes is a dynamic tool for monitoring a variety of perceptual-motor processes required when performing constructional tasks. PlayCubes is based on the ActiveCube (AC), shown in Fig 1, an electronic, Lego-like tangible user interface (TUI) designed to construct 3D objects [13]. To date, ActiveCube has been used to explore how participants used this novel interface to interact with narrative software. TSU.MI.KI is a novel toy based on the ActiveCube TUI [14], a technological enhancement of the Japanese classic tsumiki (a traditional set of wooden blocks). TSU.MI.KI allows children to actively move through a story space by physically interacting with a set of physical cubes. The TSU.MI.KI player is confronted with several tasks through a story narrative displayed on a computer screen. In order to confront the story puzzles and challenges the child needs to play with their TSU.MI.KI "magical cubes". TSU.MI.KI is realized with the ActiveCube's function of real-time shape recognition. With this function, ActiveCube has also been used as the basis for the development of Cognitive Cubes to investigate adult spatial cognitive ability [15]. The system projected a 3D virtual prototype on a large screen and the participant was asked to construct the virtual prototype using the ActiveCube physical blocks. As the participant progressed the system extracted in real-time the geometry of the structure and analyzed the similarity between the physical construction and the virtual

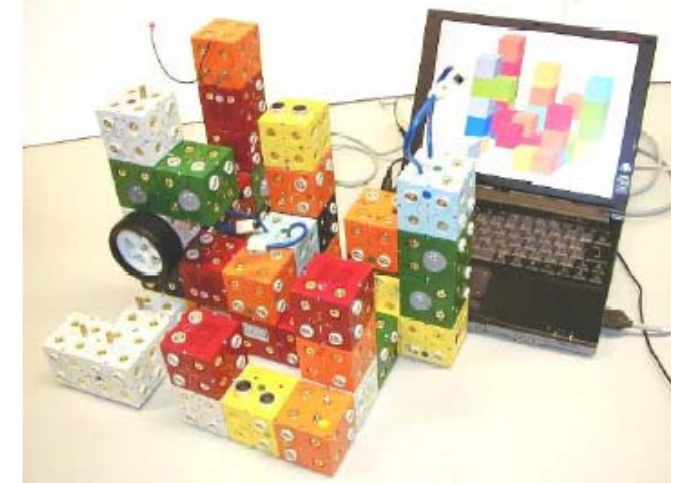

Figure 1. The ActiveCube system showing connections to run with a laptop computer. prototype. Testing showed that Cognitive Cubes were sensitive to age as well as to dementia [15].

To date, the literature does not describe any computerized tool that has been developed for assessing children's constructional ability. PlayCubes has the potential to characterize both the dynamic functional processes underlying constructional abilities as well as the actual final constructed structure. Until recently, in the absence of feasible online tools, clinicians have mainly focused on the end product of construction tasks. In the last decade diagnostic computerized tools are increasingly available in different areas. For example, digitizer-based technology is now used to analyze the writing process of children with dysgraphia [16]. The results indicated the advantages of combining both handwriting process and product testing to discriminate between children with proficient and dysgraphic handwriting.

The ActiveCube and PlayCubes systems have several advantages, which make them highly suitable for objective and automated cognitive assessment and intervention. First, they enable simulation of natural, ecologically valid environments. Second, they enable real-time, quantitative tracking the dynamic processes that may be fully documented. Third, they provide online feedback of different modalities enabling users to monitor success and failure. Finally, the game-like, computerized interaction is enjoyable, and appears to encourage cooperation and lead to heightened alertness and increase motivation. These are very important factors to be considered when assessing and treating children. The ActiveCube TUI has not yet been used as a tool to understand the dynamic functional processes underlying constructional ability in either typically developed children or in children with constructional problems. The overall goal of this research is to develop and evaluate the PlayCubes paradigm in which the ActiveCube TUI is used for the study and assessment of constructional processes among typically developed children and those with constructional problems. The specific objectives of the study presented in this paper were:

- Developing an ecologically valid environment and interface suitable for the assessment and treatment of children with constructional problems.

- Examining the feasibility and the usability of the PlayCubes TUI for typically developed children in terms of their ability to manipulate this TUI, their level of enjoyment and the effort required to perform these tasks.

- Measuring and characterizing the constructional performance of typically developed children while constructing apparatus of varying levels of complexity.

\section{METHODS}

\section{A. Participants}

The research group consisted of 38 typically developed children, age five and a half to six and a half $(\mathrm{M}$ (Mean) $=71.7$; SD (Standard Deviation) $=3.4$ ), who were recruited via a convenience sample. They had no difficulties in copying 
geometric figures, as evaluated by the Beery Developmental Test of Visual-Motor Integration (Beery VMI) [17], and no constructional problems as evaluated by the subtest from the Sensory Integration and Praxis Test (SIPT) [18]. In addition, children who were suspected of having Developmental Coordination Disorder (DCD) based on parental responses to the Children Activity Scale for Parents (ChAS-P) questionnaire [19] or who were suspected as having Attention Deficit Hyper Activity Disorder (ADHD) based on parental responses to the Conner's Parent Rating Scale-Revised-Short Form (CPRS-R:S) questionnaire [20] were excluded from the study, as were children with any neurological deficit or developmental delay.

The study was approved by the Israeli Ministry of Education Institutional Review Board. Parents of all 38 children gave their consent to participation in the study.

\section{B. Instruments}

1) Hardware: The Active Cube system, shown in Fig. 1, is an automated system for constructional cognitive assessment. ActiveCube is a set of graspable plastic cubes (the current version dimensions are $5 \mathrm{~cm}$ per edge) with magnetic rims which allow the user to physically attach or detach cubes by connecting or disconnecting their faces. Each cube is essentially a small computer which powers up and communicates with its neighbors upon connection to an adjacent cube. When children assemble a physical shape using the system they also connect a network topology which allows ActiveCube to digitize and track online the exact 3D geometry of the physical structure formed. From the user's perspective, ActiveCube is a very powerful tool; the $3 \mathrm{D}$ shape being built with it physically is tracked in the virtual domain in real-time.

ActiveCube supports a rich variety of onboard input and output devices. For example, cubes can be equipped with a gyroscopic sensor which tracks the structure's 3D orientation. Cubes can also be equipped with a light source that can be switched to illuminate them, as well as with touch sensors, vibrators, a motorized propeller and a variety of other sensors and actuators. The current ActiveCube implementation links to a host PC through a tethered cube, or base cube, which controls the network and is the first cube of each apparatus constructed with the system [21].

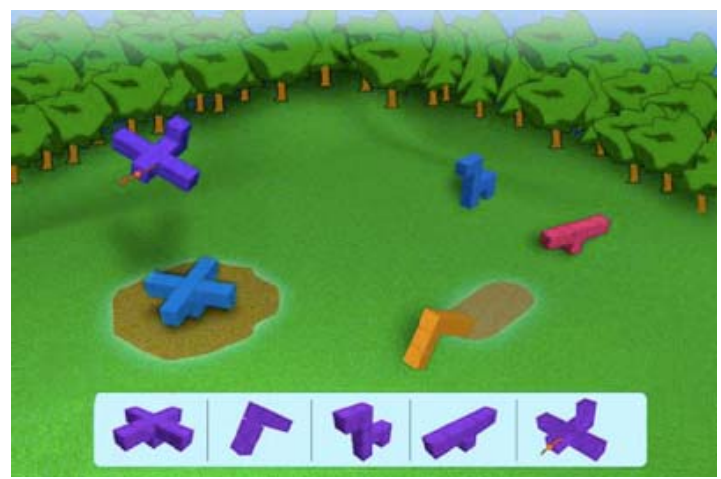

Figure 2. The playground metaphor that is used to present the five 3D playground structures: seesaw, dog, airplane, slide and carousel.

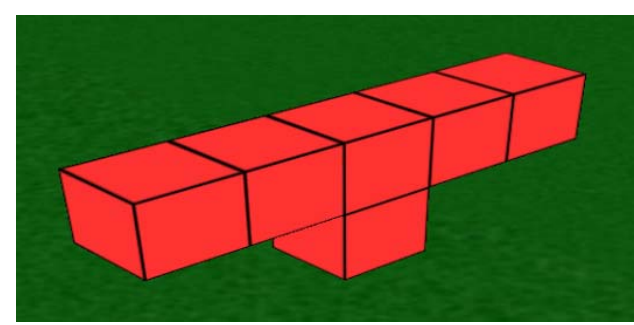

(a) seesaw

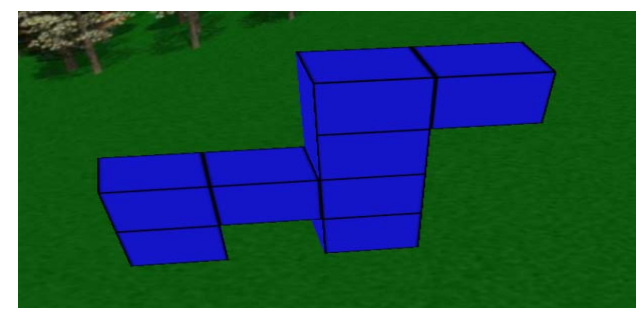

(b) $\operatorname{dog}$

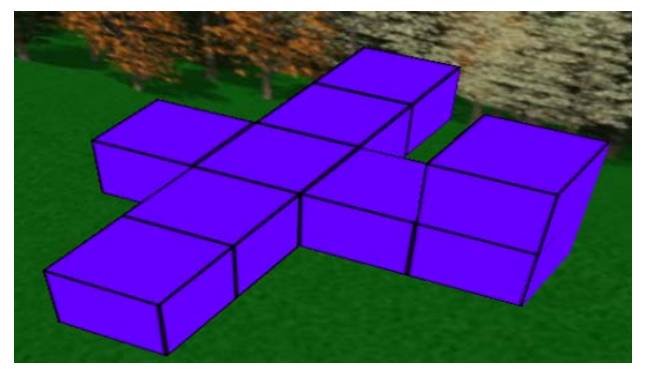

(c) airplane

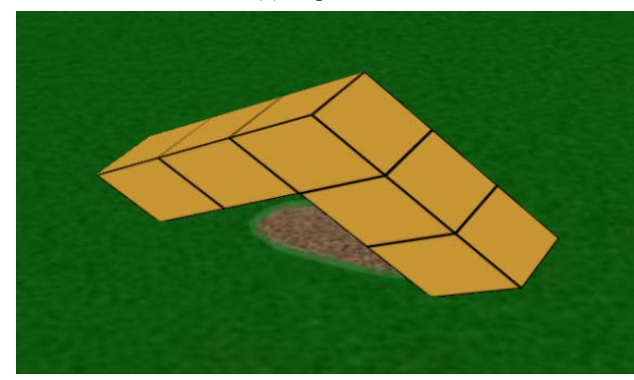

(d) slide

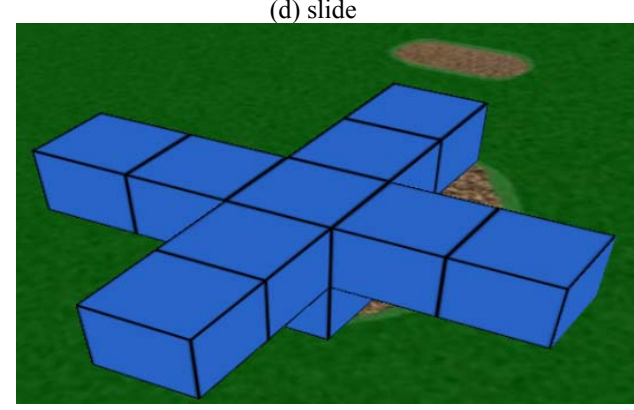

(e) carousel

Figure 3. The five 3D playground structures as presented to the children. 
2) Software: A playground metaphor, shown in Fig. 2, is used to present the PlayCubes tasks. The playground includes five 3D playground structures: seesaw, dog, airplane, slide and carousel shown in Fig 3. Each apparatus may be constructed from between five and ten cubes. The tasks for the study included:

a. Practice Task: familiarization with cubes. During this task the child learns how to connect the cubes. The tester demonstrates how to connect cubes and the child is then asked to connect a few cubes independently, receiving help if necessary.

b. Construction of a meaningful structure in the natural playground setting: The tester introduces each playground apparatus to the child. The selected apparatus is then enlarged and presented on the screen by itself. The child's task is to construct this apparatus in a "matching" strategy that we have termed "Perspective Matching". The designated apparatus is displayed in its entirety with the child viewing only the image of the structure on the screen.

c. Play: After the children finish constructing an apparatus they can play with it by manipulating the completed physical structure. The virtual apparatus on the main playground screen then mimics the actual structure's movements. Here the input/output devices of the cubes including the gyroscopic sensor, the light, the buzzer and the luminous function may provide motivating feedback. Different games were developed for each apparatus. For example, the children manipulate the dog structure to navigate through a maze with obstacles until they reach a treasure box.

\section{Outcome measures}

All sessions were recorded with a digital video recorder for subsequent viewing and analysis. A 5-point "Enjoyment Scale" was used to query each of the children following the Perspective Matching session. They were asked to rate their level of enjoyment while using the PlayCubes constructional activities. A 5-point "Effort Scale" was also used to query the amount of effort perceived by each child while performing each task. In addition they were asked about the usability of the PlayCubes and their preference for working with them versus regular buildings blocks. All connections of the ActiveCube system were recorded online for subsequent offline analysis. The following outcomes were calculated:

1) Onset time: time from the start of the task (when the designated structure is displayed on the screen) until the first cube is connected to the base cube.

2) Number of errors: error define as connecting or disconnecting of a cube which results in similarity decreasing.

3) Total connect time: time from the beginning of the task (when the designated structure is displayed on the screen) to its completion (when the last cube has been connected or disconnected.)

4) Connection time: the time taken for each step of adding well-connected cube. For example, the time taken to move from two well-connected cubes to three well connected cubes.
5) Similarity: the similarity between the designated apparatus as displayed on the screen and the apparatus as constructed by the child is used as a measure of accuracy. When the structure is not identical to the original apparatus, a score is automatically computed by the system according to the number of cubes that have been connected correctly and the number that have not been connected correctly. Equation 1 for measuring similarity was first developed and described by Sharlin et al. [15] where $i$ is an intersection of $s$ (the structure which the child built) and $p$ (the prototype), and $|i|,|s|$, and $|p|$ are the number of cubes in $i, s$ and $p$ is maximized over all possible intersections $i$ produced by rotating or translating $s$. Intuitively speaking, the algorithm computes similarity by the number of intersecting cubes minus the number of remaining "extra" cubes in the participant's structure, normalized by the number of cubes in the prototype.

$$
S=100 \cdot\left(\frac{|i|}{|p|}-\frac{|s|-|i|}{|p|}\right)
$$

\section{Procedure}

Data collection was conducted in a quiet room at various kindergartens. The children sat at a table suited to their anthropometric characteristics. The PlayCubes system and construction tasks were described to each participant at the start of the session and specific instructions in accordance with a fixed protocol were provided. At the beginning of the session there was a practice task when the child learned how to connect the cubes via demonstration. The child was then asked to connect a few cubes independently, receiving help if necessary. The child then constructed each of the five playground apparatus using the "Perspective Matching" strategy. The order of the five apparatus was predetermined. The children were instructed to press the Escape key when they had completed construction of each apparatus. The child was then given an opportunity to play with the constructed apparatus using the cubes' input/output devices to control and manipulate the virtual apparatus. This process was repeated for the next apparatus. Upon construction of all five structures, the child was asked a few questions regarding the usability of the PlayCubes and his preferences for play with PlayCubes versus regular buildings blocks, to rate his enjoyment of the task and his perceived effort using the scales described above. The whole session lasted for about 30 minutes and was performed by an occupational therapist.

\section{E. Data analysis}

Videotaped recordings of the PlayCubes construction process were used to verify that all PlayCubes connections and disconnections were correctly recorded as such by the software. Data analysis including descriptive statistics: means, standard deviations, and percentiles for all of the above three objective. To measure the differences in constructional performance between the five structures a repeated measure ANOVA was used. Post hoc comparisons were performed using the Bonferroni adjustment. 


\section{RESULTS}

\section{A. Feasibility and usability of PlayCubes}

Table I lists the children's enjoyment and effort ratings as well as their preferences for using the PlayCubes versus regular building blocks. All of the children enjoyed working with the PlayCubes. Eighty-seven percent of the children rated their use of the PlayCubes when constructing the various structures with the highest score ('enjoyed very much') of Enjoyment Scale even thought $50 \%$ of the children felt that the PlayCubes tasks required "very much effort", the highest rating on the effort scale. Overall, $95 \%$ of the children preferred to play with the PlayCubes over regular building blocks. In addition, based on observation of the children while performing these tasks, it was evident that they were motivated and maintained a high level of alertness while using the PlayCubes. They all performed the tasks in a systematic and consistent manner.

TABLE I. USER FEEDBACK (\%)

\begin{tabular}{|c|c|c|c|c|c|}
\hline & $\begin{array}{c}\text { Not at } \\
\text { all }\end{array}$ & A little & Enjoy & A lot & $\begin{array}{c}\text { Very } \\
\text { much }\end{array}$ \\
\hline Enjoyment & 0 & 0 & 5.3 & 7.9 & 86.8 \\
\hline & $\begin{array}{c}\text { Not at } \\
\text { all }\end{array}$ & Little & $\begin{array}{c}\text { Make } \\
\text { an } \\
\text { effort }\end{array}$ & A lot & $\begin{array}{c}\text { Very } \\
\text { much }\end{array}$ \\
\hline Effort & 26.3 & 18.4 & 5.3 & 0 & 50 \\
\hline \multicolumn{7}{|c|}{ Regular blocks } & \multicolumn{3}{c|}{ PlayCubes } \\
\hline & 5.3 & 94.7 \\
\hline Preference & &
\end{tabular}

All of the children succeeded in connecting and disconnecting the magnetic PlayCubes using the red strips as cues on faces of each cube. In many cases, for technical reasons, although a connection appeared to be intact mechanically, it was not recorded by the computer as being connected electronically. In all such cases of technical failure we used video monitoring to bypass the electronics and correctly register the connections and disconnections.

Fig. 4 shows the mean percent similarity scores reached while constructing each apparatus. The slide was the most difficult task (i.e., least percent similarity). This is also supported by the repeated measures ANOVA test. Since the sphericity assumption was not met as required by this test, the Greenhouse-Geisser correction was applied. The main effect of the similarity score was significant $\left(\mathrm{F}_{(2.59,95.98)}=10.03\right.$, $\mathrm{p}<0.001, \eta 2=.213$ ), Post hoc comparisons using the Bonferroni adjustment for multiple comparisons showed significant differences between the slide and the seesaw apparatus $(p=0.000)$ and between the slide and the carousel apparatus $(\mathrm{p}=0.001)$. Fig. 5 shows the percent of children who completed each task, with (blue histograms) or without (purple histograms) errors. For example, almost all of the children completed the seesaw task $(86 \%)$, and most of them completed it without any errors (68\%). Based on Fig. 5, which includes both the similarity outcome measure and the errors during construction process measure, it is evident that the seesaw was the easiest task and that the slide and carousel were the hardest tasks; most children made at least one error during these tasks and only $21 \%$ and $37 \%$ completed the slide and carousel tasks without errors, respectively.

Errors are defined as connection or disconnection of a cube which reduced its similarity to the target apparatus. Fig. 6 presents the number of errors per task. For example, on average, more than three errors were made during the slide task. During the slide task, children made more than twice as many errors than during any other task. This can be explained by the complexity of this task compared to others (number of cubes and shape of apparatus). In contrast, there were almost no errors during the seesaw task.

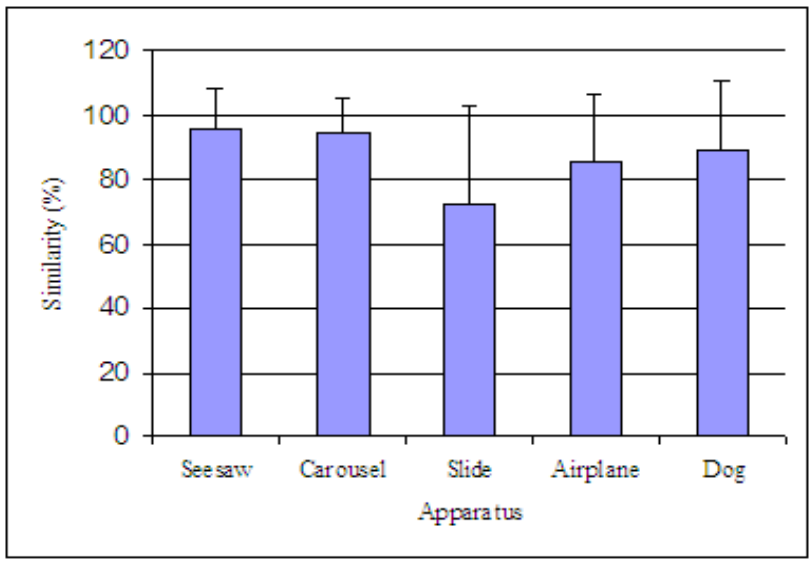

Figure 4. Similarity score

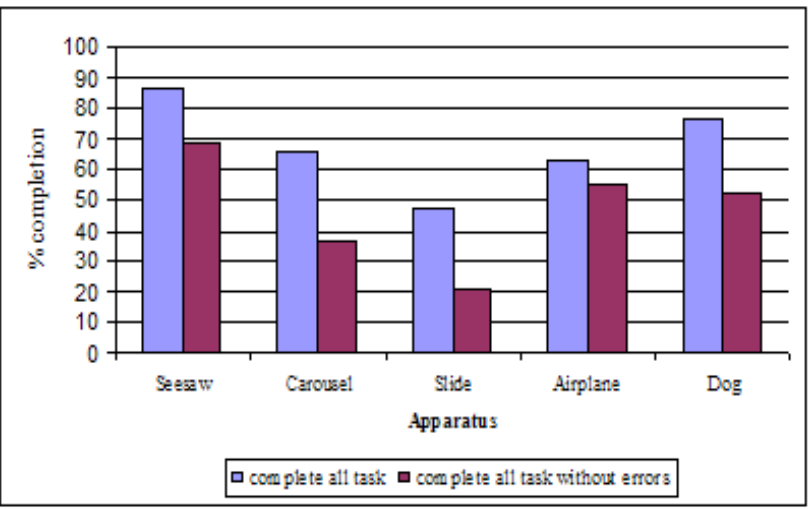

Figure 5. Completion success 


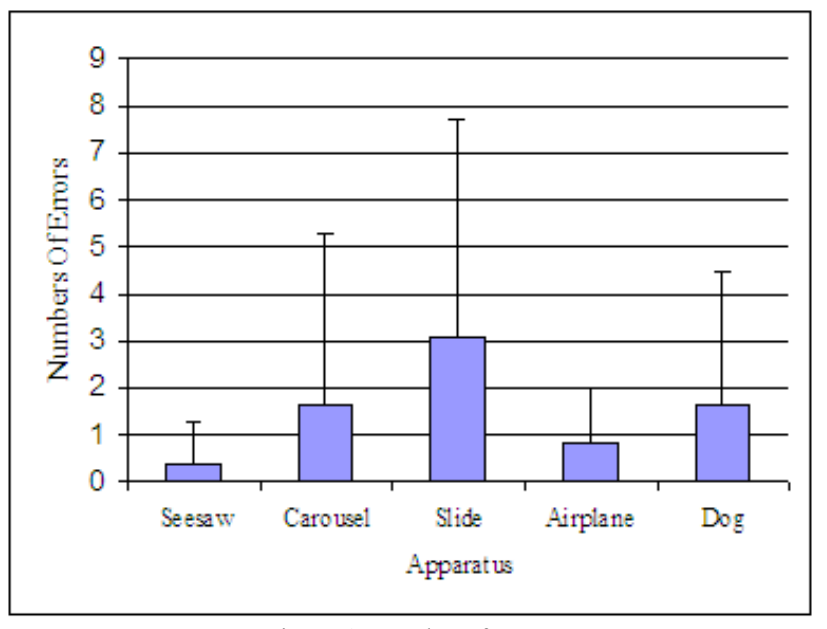

Figure 6. Number of errors

Fig. 7 and Fig. 8 describe temporal variables. Fig. 7 shows the mean time taken to complete each task. The seesaw had the fastest completion time, whereas the slide and carousel required more time than did the airplane and dog. While this information is interesting, it is not conclusive because it does not take into account the number of cubes required to complete each task and whether the task was indeed completed or not. For example, a child may complete one of the structures very quickly, but do so with considerable errors. Fig. 8 presents the mean connection time across all connections of each task for all the children. It can be seen that during the slide task, the mean connection was about twice as long as for the carousel, dog, and airplane.

Tables II and III provide a closer view of the connection time during the slide task, based on process measurements. These tables show the mean time taken for each step over all participants. The mean time for the third connection (46 s) is more than double (and even triple) that of any other connection. As shown in table III, for $55 \%$ of the children, the third connection was the hardest one; $34 \%$ of the children were not able to complete this connection, and of the $21 \%$ who completed the whole task, the third connection was the hardest one for them (based on the time it took to complete each connection).

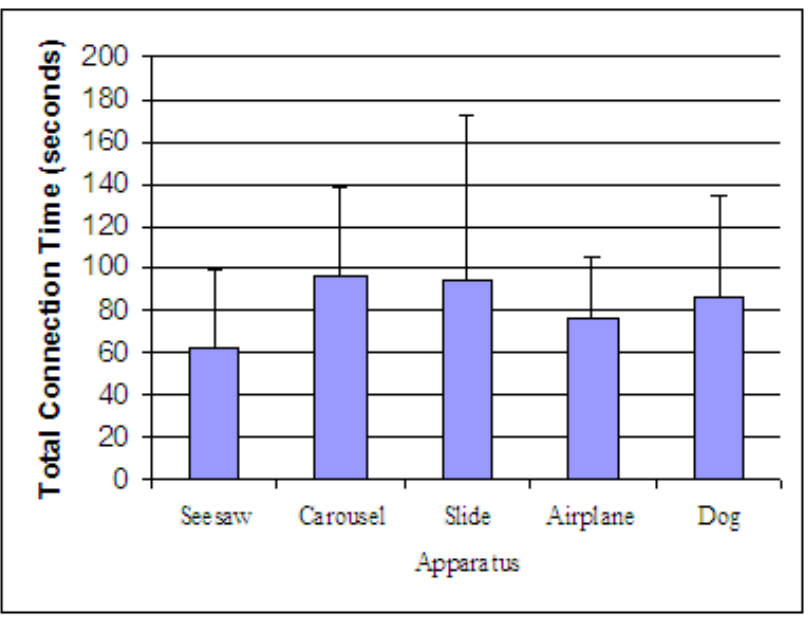

Figure 7. Total connection time

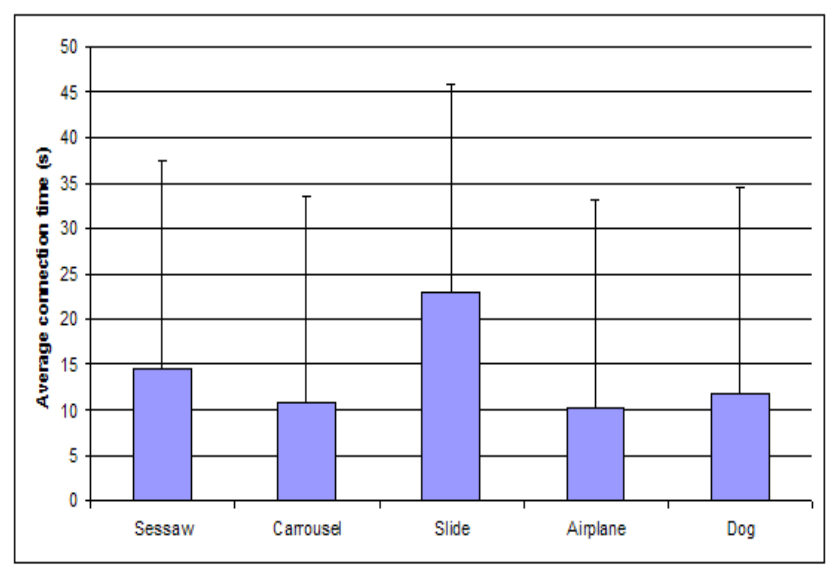

Figure 8. Mean connection time

TABLE II. CONNECTION TIME OF EACH STEP FOR THE SLIDE TASK (S)

\begin{tabular}{|c|c|c|c|c|}
\hline Connection & $\# 1$ & $\# 2$ & $\# 3$ & $\# 4$ \\
\hline Mean & 17 & 15 & 46 & 21 \\
\hline SD & 9.4 & 12 & 59 & 27 \\
\hline
\end{tabular}

TABLE III. HARDEST CONNECTION DURING SLIDE TASK (\%)

\begin{tabular}{|c|cccc|}
\hline Connection & $\# 1$ & $\# 2$ & $\# 3$ & $\# 4$ \\
\hline Did not complete & 3 & 0 & 34 & 18 \\
Longest connection time & 8 & 3 & 21 & 13 \\
\hline Hardest (sum) & $\mathbf{1 1}$ & $\mathbf{3}$ & $\mathbf{5 5}$ & $\mathbf{3 2}$ \\
\hline
\end{tabular}

Closer examination of the difficulty of each task for each participant provided additional support for these results, as summarized in Table IV. We used both outcome and process measurements to determine, for each child, the order of difficulty for each task on a 5-point scale from easiest to hardest. Specifically, we first ordered difficulty based on the percent similarity scores (Fig. 4), then adjusted this order based on the number of errors (Fig. 6) whenever the percent 
similarity score for a child was the same for more than one apparatus, and, finally, took into account the total connection time (Fig. 7) whenever the percent similarity and the number of errors was the same for more than one apparatus.

Formally, let $\operatorname{Sim}(X), \operatorname{Err}(X), T(X$, be the similarity, error num, and total connection time for task $X$, then $A$ is harder than $B$ if

$$
\begin{aligned}
& {[\operatorname{Sim}(A)<\operatorname{Sim}(B)] \quad \text { OR }} \\
& {[\operatorname{Sim}(A)=\operatorname{Sim}(B) \& \operatorname{Err}(A)>\operatorname{Err}(B)] \quad \text { OR }} \\
& {[\operatorname{Sim}(A)=\operatorname{Sim}(B) \& \operatorname{Err}(A)=\operatorname{Err}(B) \& T(A)>T(B)]}
\end{aligned}
$$

For most children the seesaw was the easiest or the second easiest task (81\%), the slide was the hardest, or second hardest task $(71 \%)$, and the carousel was Moderate-Hard (71\%). The distribution of difficulty levels for the airplane and dog apparatus was about the same.

TABLE IV. DIFFICULTY BASED ON SIMILARITY, ERRORS AND COMPLETION TIME (\%)

\begin{tabular}{|l|c|c|c|c|c|}
\hline & Seesaw & Carousel & Slide & Airplane & Dog \\
\hline Easiest & 55 & 3 & 5 & 11 & 21 \\
\hline Easy & 26 & 13 & 11 & 26 & 21 \\
\hline Moderate & 3 & 45 & 11 & 16 & 26 \\
\hline Hard & 8 & 26 & 21 & 21 & 21 \\
\hline Hardest & 5 & 11 & 50 & 24 & 8 \\
\hline
\end{tabular}

\section{INITIAL DISCUSSION, CONCLUSIONS AND FUTURE RESEARCH}

The results of this study were highly positive in terms of verifying the participants' interest in and enjoyment of the PlayCubes playground paradigm. The participants understood the tasks and were motivated to carry them out. This is reinforced by the fact that half of the children found that the tasks were very difficult. Almost all of the children $(95 \%)$ preferred to play with the PlayCubes over regular building blocks.

In addition to providing a summary score for the output measures, the results indicate that PlayCubes provide the ability to quantitatively track constructional processes when performing various building tasks. They provide more accurate information about the strategy that a child uses for constructional tasks. For example, data demonstrated that for the slide task the major problem occurred during the attempt to add the fourth cube. This is enlightening in view of the geometry of this structure which entails an awkward change in direction at this point (cf. Fig. 3). The data also demonstrated that during the slide and carousel tasks, about half of the children made errors but were able to correct them and complete the rest of the task.

It was clearly seen that there are differences in the level of difficulty between the five structures although some of differences were not statistically significant, perhaps due to the small sample size or the fact that the children who participated in the study have a normal constructional ability.
The most difficult apparatus to construct was the slide, even though it has the least number of cubes. We find this contrast between the apparent simplicity of the task and the difficulty in performing it in practice to be very intriguing. The reason for that, we believe, is the different orientation of the slide compare to the rest of the structures, which were aligned with the $x-y$ cartesian plane. These assumptions are supported by the fact that most of the children did not succeed in connecting the fourth cube in the slide. In addition, for the carousel apparatus the lower support cube was difficult for many of children. This may be due to the fact that it was partially hidden.

In summary, the findings show that the PlayCubes paradigm appears to be able to monitor the constructional processes in depth, while providing a final output result when performing constructional tasks. The results show that the PlayCubes system and the playground apparatus tasks appear to be sensitive to differences in the constructional abilities of the children and were very engaging and motivating for the study participants. In common with other TUIs such as TSU.MI.KI, Cognitive Cubes, and Triangles, a flat TUIs which allow interactive construction of 2D shapes [22], the PlayCubes have the potential to enhance our understanding of visuospatial processes during concrete constructional activity. Future work is being directed towards testing the use of the PlayCubes to assess children with constructional difficulties as well as to use them as an intervention tool. Our next steps will focus on:

1. Examining the feasibility and the usability of the PlayCube TUI for children with constructional problems in terms of their ability to manipulate this TUI, their level of enjoyment and the effort required to perform these tasks.

2. Measuring and characterizing the performance of children with constructional problems while constructing apparatus of varying levels of complexity.

3. Determining whether the PlayCube TUI is sensitive to performance differences between children with constructional problems and typically developed children.

4. Developing an ecologically valid environment and interface suitable for the training/treatment of children with constructional problems.

5. Evaluating the effectiveness of the PlayCube TUI as a training/ treatment tool for children with constructional problems.

6. Measuring and characterizing the constructional performance of other population such as adults or children with perceptual, cognitive or fine motor deficits due to neurological disease.

7. Continuing to improve the ActiveCube system via the use of smaller cubes, and wireless connections, probably based on Bluetooth technology. Such improvements will enable building more complex structures, with more cubes.

\section{REFERENCES}

[1] J. S. Fisher and D. W. Loring, "Construction," in Neuropsychological Assessment, M. D. Lezak, D. B. Howieson, and D. W. Loring, Eds ,4th ed. New York: Oxford University Press, 2004, pp. 531-568. 
[2] K. Fernando, L. Chard, M. Butcher, and C. Mckay, "Standardisation of the Rey Complex Figure Test in New Zealand children and adolescents," NZ. J. Psychol, vol.32, pp. 33-38, 2003.

[3] D. Wechsler, Manual for Wechsler Preschool and Primary Scale of Intelligence, 3rd ed. (WPPSI-III). New York: Psychological Corporation, 2002.

[4] S. Cahan, Manual for the WISC-R95. Jerusalem: Ministry of Education, The Psychological and Counseling Service, 1998.

[5] D. Wechsler, Manual for the Wechsler Adult Intelligence Scale, 3rd ed. (WAIS-III). San Antonio: The Psychological Corporation, 1998.

[6] N. Katz, S. Parush, and R. Traub Bar-Ilan, The Dynamic Occupational Therapy Cognitive Assessment for Children (DOTCACh) Manual. Pequannock NJ: Maddak Inc, 2005.

[7] R. Sternberg, "Intelligence and lifelong learning: What's new and how can we use it?" American Psychologist, vol. 52, no. 10, pp. 1134$1139,1997$.

[8] A. A. Rizzo, and J. G. Buckwalter, "Virtual Reality and Cognitive Assessment and Rehabilitation: The State of the Art," in Virtual Reality in Neuro-Psycho-Physiology: Cognitive, Clinical and Methodological Issues in Assessment and Rehabilitation, G. Riva, Eds. Amsterdam: IOS Press, 1997, pp. 123-146.

[9] A. A. Rizzo, and J. G. Buckwalter, "Special Issue: Virtual Reality Applications in Neuropsychology Guest Editor's Introduction," Presence: Teleoperators and Virtual Environments, vol. 10, no. 4, 2001.

[10] E. Sharlin, B. Watson, Y. Kitamura, F. Kishino, and Y. Itoh, "On tangible user interfaces, humans and spatiality," J. Pers. Ubiquitous. Comput, Vol.8, no.5, pp. 338-346, 2004.

[11] G. Groth-Marnat, Handbook of Psychological Assessment, 3rd ed. New York: John Wiley \& Sons, 1997.

[12] A.A. Rizzo, and G. Kim, "A SWOT analysis of the field of virtual rehabilitation and therapy," Presence:Teleoperators and Virtual Environments, vol. 14, no. 2, pp. 1-28, 2005.
[13] Y. Kitamura, Y. Itoh, and F. Kishino, "Real-time interaction with ActiveCube," in Proc. Of Conference on Human Factors in Computing Systems. Seattle, Washington, USA, March 2001.

[14] Y. Itoh, S. Akinobu, H. Ichida, R. Watanabe, Y. Kitamura, and F. Kishino, "TSU.MI.KI: stimulating children's creativity and imagination with interactive blocks," Second Int'l Conf. of Creating Connect Collab Comput, pp. 62-70, 2004.

[15] E. Sharlin, Y. Itoh, B. Watson, Y. Kitamura, L. Liu, S. Sutphen, "Cognitive cubes: a tangible user interface for cognitive assessment," in Proc. of the conference on Human Factors in Computing Systems. Minneapolis, USA, April 2002.

[16] S. Rosenblum, P. L. Weiss, and S. Parush, "Handwriting evaluation for developmental dysgraphia: Process versus product.," Reading and Writing: An Interdisciplinary Journal, vol 17, pp. 433-458, 2004.

[17] K. E. Beery, N. A. Buktenica, and N.A. Beery, The Beery-Buktenica Developmental Test of Visual-Motor Integration, 5th ed. Lutz, FL: Psychological Assessment Resources, 2003.

[18] A. J. Ayres, Sensory integration and praxis test. Los Angeles: Western Psychological Services, 1989.

[19] S. Rosenblum, "The development and standardization of the Children Activity Scale (ChAS-P/T) for the early identification of children with Developmental Coordination Disorders," Child: Care, health and development, vol. 32, no. 6, pp. 619-632, 2006.

[20] K. G. Conners, Conners' rating scales-revised manual. New York: Multi-Health Systems, Inc.CPRS questionnaire, 1997.

[21] R. Watanabe, Y. Itoh, M. Asai, Y. Kitamura, F. Kishino, and H. Kikuchi, "The soul of ActiveCube-implementing a flexible, multimodal, three-dimensional spatial tangible interface," Computers in Entertainment, vol. 2, no. 4, 6b , 2004.

[22] M. Gorbet, M. Orth, and H. Ishii, "Triangles: Tangible interface for manipulation and exploration of digital information topography," Conference on Human Factors in Computing Systems, pp. 49-56, 1998. 